\title{
Theorists reject challenge to standard model
}

David Adam

Reports of the death of the standard model of particle physics are highly exaggerated, a group of theoretical physicists asserted last week. They questioned recent claims about experimentalists' results that appear to violate the 40-year-old standard model of matter and forces.

A blast of media speculation followed last month's announcement that scientists at the Brookhaven National Laboratory in New York state had obtained data that disagreed with the theoretical result predicted by calculations based on the standard model (see Nature 410, 28-29; 2001).

The group used the world's largest superconducting magnet - the muon storage ring — to help to measure the magnetic moment of particles called muons, which shows how the particles behave in a magnetic field. They say their results are different enough from predicted values to infer physics that the standard model cannot explain.

The press release announcing the finding was headed: "Physicists announce possible violation of Standard Model of particle physics", setting a tone that was quickly echoed by the international press.

At first, sceptical physicists pointed out that the difference lacked the statistical significance normally required in particle physics. But now some theorists charge that the Brookhaven group has been comparing its result with the most convenient theoretical value for the muon's magnetic moment. The sceptics say there are other predicted values for it which are "equally legitimate" and which would be compatible with the new experimental data.

\section{Loaded dice}

"These experimentalists are playing with loaded dice," claims Francisco Ynduráin, a theoretical physicist at the Independent University of Madrid. Ynduráin placed a critique of the Brookhaven claim on the Los Alamos preprint server (http://xxx.lanl.gov/ format/hep-ph/0102312) in February.

“They've chosen the one theoretical calculation that disagrees with their result so as to claim they've discovered something new," Ynduráin says. In fact, he contends, the new experimental data appear to validate the other theoretical values, which broadly agree with one another.

Lee Roberts, a physicist at Boston University and spokesman for the Brookhaven experiment, robustly denies that the team cherry-picked convenient predicted values for comparison.

"The one we used is the latest and what we think is the best value," he says. "I've followed this for over ten years and the [theoretical predictions] have steadily got more precise as

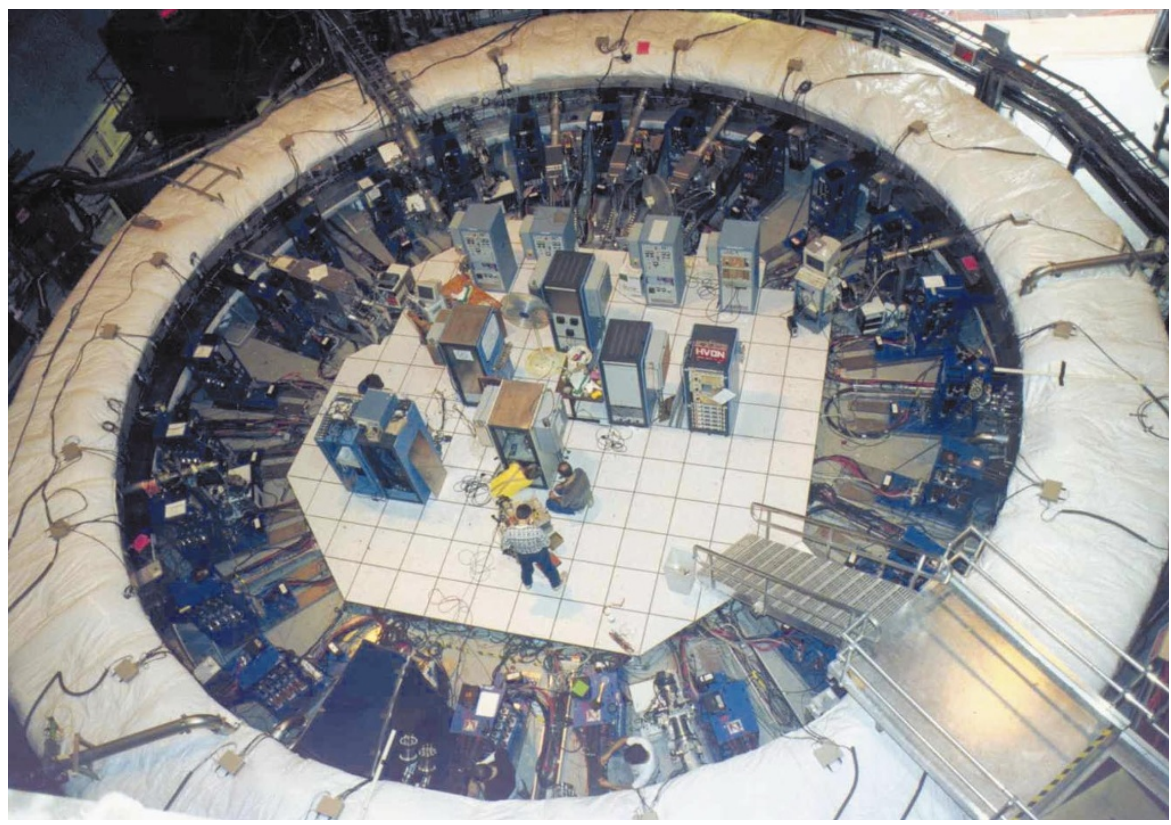

Ring of confidence: but how valuable is the result obtained from Brookhaven's muon experiment?

additional data have been included. I look at the others and I say they are old numbers."

The theoretical values at issue come from a complex set of equations used to predict how rapidly muons wobble in a magnetic field, taking account of the influence of other subatomic particles around them. To do this, theoretical physicists use data both from previous experiments and the mathematical framework of the standard model, which seeks to explain how quarks, neutrinos, electrons, muons and other particles interact.

Roberts says that the accuracy of the theorists' prediction inevitably improves as more experimental data are fed into the equation. But does this mean that the framework underpinning the theorists' work is wrong, if their latest prediction is disproved by an experimental result? Ynduráin, who devised one of the earlier predictions, says not.

"Later is not necessarily better," he says, explaining that each calculation places slightly different emphasis on the components making up the equation and their relative errors. Since he posted his objections, he says that "dozens" of physicists have contacted him to agree.

One of these is Valentine Telegdi, a highenergy physicist at the California Institute of Technology. He says the argument that the most recent theoretical treatment is also the most thorough is "not compelling". And he repeats physicists' initial concerns about the statistical significance of the result.

"The departure from the standard model, even with the theoretical prediction favoured by the Brookhaven group, is statistically weak," says Telegdi. Statistically, the gap between the theoretical and the experi- mentally obtained results is 2.7 standard deviations, where experimentalists normally like to have three standard deviations before declaring a result. The other theoretical values are about one standard deviation away from the experimental result, which is not statistically significant at all.

\section{Issue not settled}

"In my opinion the issue is certainly not settled," says Martinus Veltman, a high-energy physicist based at the University of Michigan, who won the Nobel Prize in Physics in 1999. "The theory and the data used as input for the theory are too complicated to make things as cut and dried as one would want. I tend to agree with Ynduráin that just comparing with the most recent analysis is somewhat too simplistic."

But other physicists support the Brookhaven team's position. They agree that the theoretical prediction based on the most up-to-date information must be the one that best reflects the standard model.

Although accepting that there is "not yet a consensus of opinion", Gordon Kane, a physicist at the University of Michigan, says: "We have to make a choice, and there is a tentative agreement to use [the most recent value] until there is something better."

Kane and Roberts point to new experimental data that should be available within months. These should help both to rework the predicted value and to test the robustness of the Brookhaven results further. If, as Roberts expects, it supports his group's findings, Kane says the implications could be "far more powerful than even optimists are claiming right now". 